- Qualitative and quantitative data was analysed on day $1,4,8,12$;

- The amount and ingredients were standardised, and a doctor stated dosage and frequency on the patient drug chart;

- Dryness was scored from 1-10 (1 least -10 highest), and to state any comments.

Results Introducing the mouthwash has had a positive impact on patient care. 82 patients were referred across the services. Inpatients 49, Day hospice 9, Out patients 24 . $83 \%$ of the 82 participants continued stating positive comments compared to $17 \%$ who had to withdraw from the study. The average dryness score decreased from 7.4 start day 1 to 3.6 day 12 . Positive comments stated, 'Helps remove the metal taste after chemotherapy'; 'used at night don't have a dry mouth in morning'; 'helped after using inhaler'; 'refreshing better than others I have used' and 'if used before meals able to eat without feeling dry'.

Conclusion The results from patient feedback were qualitative and quantitative. Due to the volume of patients referred nurses were trained to prepare the mouthwash. Several hospices, care homes and the local hospital have shown an interest in this QIP due to the benefits patients expressed and want to use it for their patients. The positive impact for this change for our patients mean that we will continue to use the essential oil mouthwash.

\section{P-133 MOUTH CARE MATTERS: RAISING AWARENESS OF THE IMPACT OF DENTURE LOSS}

Connie Gillman, Victoria Cowell, Diana Lees. Mid Cheshire Hospitals, Crewe, UK

\subsection{6/bmispcare-2019-HUKNC.156}

Background Patients who rely on dentures do so not only for eating and communication but also as part of their identity and self-esteem. In 2017, denture losses in hospitals were estimated at 9,500 by the British Dental Association. This project was devised following the death of a patient who had lost her dentures during her final illness in hospital, which impacted upon her ability to eat and to communicate with her family and healthcare professionals.

Aims To assess the impact loss of dentures has on patients and to increase awareness amongst clinical staff regarding difficulties encountered by these patients.

Method A questionnaire was given to patients with dentures across two medical wards, inquiring:

- How loss of dentures would impact upon them?

- Had they been provided with a denture pot?

A 'Denture Reminder Form' was provided for attachment to the admission documentation with the aim of improving the use of denture pots.

Discussion

- $38 \%$ of patients with dentures were offered a denture pot, falling to $16 \%$ after implementation;

- $95 \%$ of patients felt it impacted their ability to communicate and $79 \%$ on their ability to eat;

- In 2018, 7 claims for replacement dentures were made costing $£ 969$.

Currently, the Trust has no standardised admission documentation making it difficult to ensure the form is regularly used, and there were instances of the form being discarded completely.
Healthcare professionals witness the impact on patients' quality of life, but have not recognised a simple intervention which can reduce denture losses together with the 'Mouth Care Matters' campaign (https://mouthcarematters.hee.nhs.uk/).

Conclusion We have 'one chance to get it right' (Department of Health, 2015) and for patients at the end of their lives, maintenance of their self-identity, self-esteem and ability to communicate and eat is as important as the medical care they receive. There are improvements that can and must be made to promote good oral care at the end of life.

\section{P-134 SUBCUTANEOUS LIDOCAINE INFUSION FOR COMPLEX PAIN IN HOSPICE INPATIENTS - A PRELIMINARY REPORT}

Jasmine Lee, Emma Hall, Rob George. St Christopher's Hospice, London, UK

\subsection{6/bmjspcare-2019-HUKNC.157}

Background Neuropathic pain is common and challenging to treat. Systemic lidocaine, a local anaesthetic, has established use in pain management (Eipe, Gupta \& Penning, 2016). Intermittent lidocaine infusions are safe, better than placebo and equally effective as other analgesics such as morphine (Challapalli, Tremont-Lukats, McNicol, Lau et al., 2005). Anecdotes of subcutaneous lidocaine infusions also report effectiveness in some cancer patients (Seah, Herschtal, Tran, Thakerar et al., 2017). To our knowledge use in palliative care patients has not been reported.

Aims

- To describe and evaluate subcutaneous lidocaine infusions in hospice inpatients;

- To assess from patients' and clinicians' observations if lidocaine is feasible, potentially effective, safe and tolerable.

Method Sequential retrospective review of hospice inpatients over seven months (November 2018 to May 2019) given subcutaneous lidocaine infusion for refractory pain. Patients were given a $2 \%$ lidocaine dose per 24 hours by CSCI. IPOS scores and documentary text were analysed at day 0,1 and 3 .

Results Twelve patients (eleven with malignancy) received lidocaine. All had complex pain, strong opioids and co-analgesia. Eight required anaesthetic intervention.

In seven pain scores improved. Drowsiness improved in seven. Two with worsening pain were close to death however, benefited in alertness. Overall two showed no improvement in either pain or drowsiness.

All tolerated lidocaine well for periods up to 122 days (median 7). One stopped prematurely due to incidental sepsis. One experienced opioid toxicity after dose increase which may be ascribed to lidocaine effects. One continued lidocaine at home and for $>4$ months.

Conclusions In this preliminary report, for patients with complex pain, refractory to standard multidrug regimes, lidocaine is a well-tolerated, safe, potential option for hospice inpatient use and supports the available anecdotal evidence. Observations suggest benefits in pain control and quality of life.

Lidocaine appears particularly valuable

- where patients are experiencing drowsiness from high frequency or high dose opioids;

- as an option pending anaesthetic intervention and;

- inpatients unable to swallow conventional neuropathics.

These findings justify a formal study which is under development. 